\title{
Effect of the type of oak barrels employed during ageing on the ellagitannin profile of wines
}

\author{
I. GARCÍA-ESTÉVEZ Đ, M.T. ESCRIBANO-BAILÓN Đ, J.C. RIVAS-GONZALO @ and C. ALCALDE-EON \\ Grupo de Investigación en Polifenoles (GIP), Unidad de Nutrición y Bromatología, Facultad de Farmacia, University of Salamanca, \\ E37007 Salamanca, Spain \\ Corresponding author: Professor Maria Teresa Escribano-Bailón, email escriban@usal.es
}

\begin{abstract}
Background and Aims: Ellagitannins released from wood to wine during barrel ageing can affect wine colour and astringency. This work aimed to examine the influence that oak wood type (French or American) and age (first-fill or second-fill) of barrels has on the concentration of individual ellagitannins and their evolution during winemaking and ageing.

Methods and Results: The ellagitannin composition of red wines during ageing was determined by HPLC-MS/MS-multiple reaction monitoring. Differences in the extractability and stability of the individual ellagitannins were observed. Moreover, the ellagitannin composition of wines was determined by the type of barrel. Wines aged in new French oak barrels showed a concentration of ellagitannin about twofold higher than that aged in American or in second-fill French ones. The vescalagin/castalagin ratio was higher in wines aged in French oak barrels than that in wines aged in American barrels. Wine samples were grouped by discriminant analysis into three groups depending on the type of barrel employed during ageing.

Conclusions: The ellagitannin profile of aged red wines is strongly influenced by the type of oak barrel employed. Wines aged in new French oak barrels can be differentiated from those aged in second-fill French or in new American oak barrels by the ellagitannin concentration, whereas the vescalagin/castalagin ratio may assist in tracing the oak type (French or American) of the barrels.
\end{abstract}

Significance of the Study: The ellagitannin concentration along with the vescalagin/castalagin ratio in red wines aged in oak barrels can be employed for tracing the oak type of the barrels.

Keywords: discriminant analysis, ellagitannin, French and American oak barrel, HPLC-MS/MS-MRM, red wine, second-fill oak barrel

\section{Introduction}

Red wines are normally aged in oak wood barrels in order to improve their overall sensory attributes. During this maturation process, the wine undergoes many sensory changes as a consequence of the reactions between wine components, the oxygen that slowly diffuses through the oak barrel and the compounds released from wood (Puech et al. 1999, Glabasnia and Hofmann 2006). Ellagitannins (Figure 1) are among the oak wood compounds that can participate in oxidation reactions and consequently affect some relevant wine sensory properties, such as colour and astringency, either by favouring the polymerisation reactions between flavanols and/or anthocyanins (Timberlake and Bridle 1976, Vivas and Glories 1996) or by directly reacting with them leading to acutissimins or anthocyanin-ellagitannin hybrid pigments (Quideau et al. 2005, Jourdes et al. 2009, García-Estévez et al. 2013). Moreover, because of their ability to bind proteins (Hofmann et al. 2006), ellagitannins can directly affect wine astringency (Hofmann et al. 2006, Stark et al. 2010, Chira et al. 2015). It has been reported that the astringency induced by ellagitannins appears to be different from that induced by flavanols, being described as a rather mellow (Chira et al. 2015) and smooth astringency with a velvety mouth-coating sensation (Stark et al. 2010), with an impact in the roundness and amplitude of the red wines (Michel et al. 2011). The concentration of ellagitannin in barrel-aged wines is directly conditioned by that in the staves of the oak barrels (Michel et al. 2011), which, in turn, is conditioned by the oak species from which they are manufactured. Among the three oak species traditionally used in cooperage, the wood of the American oak (Quercus alba L.) has a concentration of ellagitannin (from 3 to $8 \mathrm{mg} / \mathrm{g}$ of dry wood) lower than that of the French oaks. Among the French oaks, Quercus robur L. wood shows the highest concentration (from 15 to $48 \mathrm{mg} / \mathrm{g}$ of dry wood) whereas that of the wood obtained from Quercus petraea (Matt.) Liebl. is intermediate (from 8 to $34 \mathrm{mg} / \mathrm{g}$ of dry wood) (Scalbert et al. 1988, Masson et al. 1995, Chatonnet and Dubourdieu 1998, Prida and Puech 2006). Other factors, such as the age and the geographical origin of the tree (Masson et al. 1995, Fernández de Simón et al. 1999, 2003) and the type and length of the drying and toasting periods (Masson et al. 1995, Cadahía et al. 2001) can also influence the ellagitannin concentration in the staves from which the barrels are made. Nevertheless, the concentration found in wines is lower than that which could be expected from the concentration in wood. The different extractability (García-Estévez et al. 2015) and reactivity (Quideau et al. 2005, Jourdes et al. 2009) of the individual ellagitannins influences the concentration in wines (Puech et al. 1996). As a result, the proportion of the individual ellagitannins may change in wine respecting those observed in wood (Michel et al. 2011).

For all these reasons, it is of interest to determine the concentration of the ellagitannin and individual ellagitannins in wine and how they evolve during winemaking and ageing. In addition, French oak barrels are more costly than American 


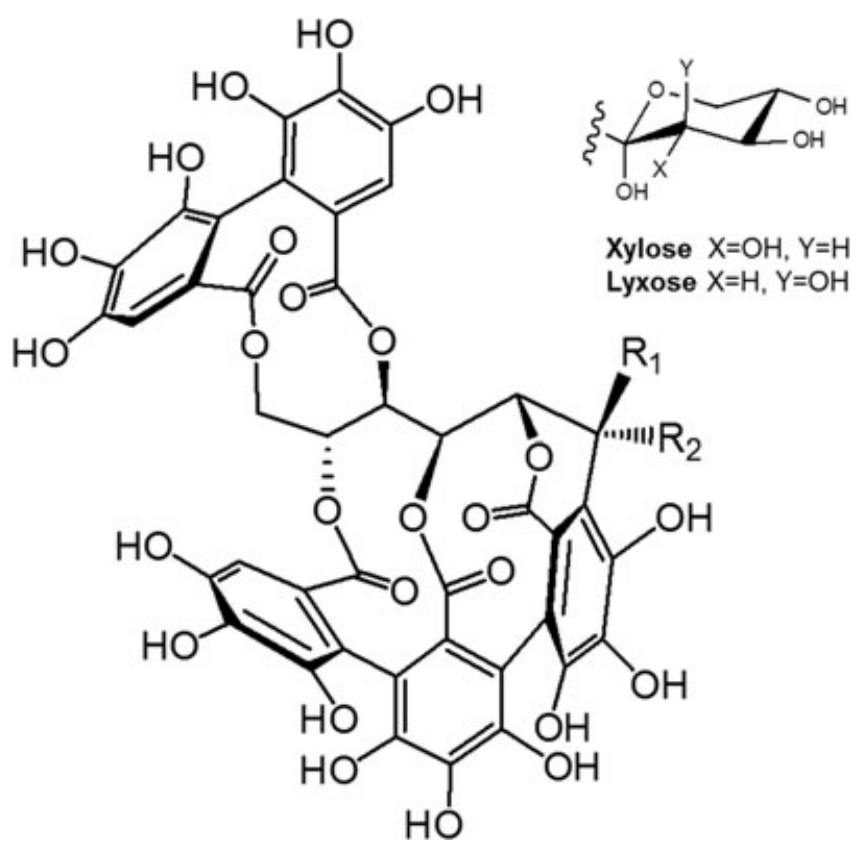

Figure 1. Structure of the main oak ellagitannins: castalagin $\left(R_{1}=H, R_{2}=O H\right)$, vescalagin $\left(R_{1}=O H, R_{2}=H\right)$, grandinin $\left(R_{1}=\right.$ lyxose, $\left.R_{2}=H\right)$ and roburin $E$ $\left(R_{1}=\right.$ xylose, $\left.R_{2}=H\right)$.

oak barrels and second-fill French barrels, and thus, a comparative study of the ellagitannin concentration and profiles in these types of oak barrel is useful. Few studies have focussed on this subject, and most of them have analysed the detailed ellagitannin composition at only one stage of the ageing period (Glabasnia and Hofmann 2006, Michel et al. 2011) or have quantified the ellagitannin concentration as the ellagic acid released after hydrolysis of the ellagitannin present in the wine (Michel et al. 2011). Jourdes and co-workers (2011) have studied the evolution of the individual ellagitannins during ageing but only in wines aged in French oak barrels. Hence, the aim of this work was to study the influence that the oak wood type (French or American) and age (first-fill or second-fill) of the barrels can exert on the concentration of the ellagitannin and individual ellagitannins and on their evolution during winemaking and ageing under winery conditions. In addition, the influence on the concentration of ellagitannin of some winery operations that affect, in turn, the oxygen content of wine (e.g. racking or bottling) has also been studied. Furthermore, the present study also aimed at confirming under commercial winery conditions some of the conclusions drawn about the extractability of individual ellagitannins in model systems (García-Estévez et al. 2015).

\section{Materials and methods}

\section{Chemicals}

Grandinin, vescalagin, roburin E and castalagin were extracted and isolated (purity $>95 \%$ ) from medium-toasted chips of Q. petraea (Matt.) Liebl. wood (García-Estévez et al. 2010). The identity of the pure ellagitannins was assigned from the data obtained in the HPLC-diode array detectorMS and in ${ }^{1} \mathrm{H}$ and ${ }^{13} \mathrm{C}$ nuclear magnetic resonance analyses (García-Estévez et al. 2010) and compared with that in the literature (Glabasnia and Hofmann 2006; Fridrich et al. 2008). (-)-Gallocatechin was purchased from Sigma-Aldrich (St Louis, MO, USA), and solvents (analytical grade) were purchased from Prolabo (Briare, France).

\section{Experimental design}

Vitis vinifera L. cv. Tempranillo grapes for winemaking were harvested from a vineyard located in the Spanish AOC Ribera de Duero region (La Horra, Burgos, Spain). Winemaking and ageing were performed under commercial winery conditions. The alcoholic fermentation/maceration step was carried out in a French oak wooden tank (12 $500 \mathrm{~L})$. Once this step was finished, the wine (wine Vl) was transferred into three types of oak barrels (two $225 \mathrm{~L}$ barrels for each type of oak wood) in which malolactic fermentation and ageing took place. To be precise, two new (F) and two second-fill (S) barrels made from French oak wood (Q. petraea) and two new barrels made from American (A) oak wood (Q. alba) were employed. The oenological parameters of the wine at the time of its transfer to the oak barrels were as follows: alcohol $14 \% \mathrm{v} / \mathrm{v}$; $\mathrm{pH} 3.7$; TA (milliequivalents of tartaric acid) $3.7 \mathrm{~g} / \mathrm{L}$; and total polyphenol index 83. All barrels were manufactured by Societé Seguin Moreau, Merpins, Poitou-Charentes, France, from naturally seasoned (24 months), medium-toasted oak wood. After barrel ageing, all wines were bottled. Wines were sampled at several stages of winemaking and of barrel and bottle ageing (Table 1). Similarly to what is performed in commercial wineries in order to homogenise wines, samples taken from the same type of barrels were blended at each sampling point. Then, each blended sample was fractionated into duplicates and analysed by HPLC-electrospray ionisation (ESI)-MS/MS-multiple reaction monitoring (MRM).

One possible drawback of working with samples subjected to commercial winemaking was that only one initial wine was submitted to the ageing process. For this reason, the same study was carried out simultaneously in the same winery with another wine (wine V2, alcohol, 13.9\% v/v; pH 3.6; TA 3.4 g/L; and total polyphenol index 84) that was made from the same cultivar of grapes harvested from the same vineyard and made following the same procedure as the first wine. The only difference between the two wines was the tank employed for alcoholic fermentation and maceration steps; for wine Vl, it was second-fill and new for wine V2. Thus, with these two wines, the number of samples from each type of barrel ageing (F, A and S barrels) was increased.

\section{HPLC-ESI-MS/MS-MRM analysis of ellagitannins}

The concentration of ellagitannins in the wine samples was determined by a two-step process. First, the samples were fractionated in duplicate following a two-step fractionation

Table 1. Sampling points and their corresponding stages of winemaking and ageing.

\begin{tabular}{lll}
\hline Sampling point & Wine container \\
\hline 0 & Devatting & Oak tank $\dagger$ \\
1 & MLF & Oak barrels (F, A and S) \\
2 & End of MLF & \\
3 & Before 1st racking & \\
4 & 7 days after 1st racking & \\
5 & Before 2nd racking & \\
6 & 7 days after 2nd racking & \\
7 & 7 days after 3rd racking & Bottle \\
8 & 3 months after bottling & \\
9 & 6 months after bottling & \\
\hline
\end{tabular}

†All alcoholic fermentations took place in French oak tanks. A, new American oak barrels; F, new French oak barrels; MLF, malolactic fermentation; S, second-fill French oak barrels. 
method previously developed in our laboratory (García-Estévez et al. 2010). Second, (-)-gallocatechin was added as an internal standard $(15 \mathrm{mg} / \mathrm{L})$ to the ellagitannin-rich fraction, which was then analysed by HPLC-ESI-MS/MS-MRM. The HPLC was a Hewlett-Packard 1100 series LC (Agilent Technologies, Waldbronn, Germany) and the method had previously been developed in our laboratory (García-Estévez et al. 2010). The MS was an API 3200 Qtrap equipped with an ESI source and a triple-quadrupole linear ion trap mass analyser controlled by Analyst 5.1 software (Applied Biosystems, Darmstadt, Germany). Mass conditions have been previously optimised and validated for the analysis of oak ellagitannins (García-Estévez et al. 2012). An MRM analysis in negative mode was employed to detect the transitions (each parent ion-daughter ion pair) corresponding to the analysed ellagitannins [castalagin (933/631), vescalagin (933/301), and grandinin and roburin E (1065/249)] and to the internal standard [gallocatechin (305/125)]. One calibration curve for each of the ellagitannins was prepared by plotting the ratio between the signal of the transition of each ellagitannin at different concentration values and that of the transition of the internal standard versus the ellagitannin concentration. These calibration curves were then used to quantify these four ellagitannins in the ellagitannin-rich fraction of all the wine samples after the addition of the internal standard.

\section{Statistical analysis}

A supervised chemometric tool was employed to find if, from the data corresponding to ellagitannin concentration, it is possible to classify the different wines samples into predefined groups, to determine how well classified they are and what causes this separation. Specifically, canonical discriminant analysis was used for data analysis as a method for multi-group discrimination (Brereton 2003). This methodology employed a stepwise feature selection to choose the most significant variables for the discrimination between classes using $F$-statistic to test the significance of the change in Wilks' lambda by adding or removing a variable. The dataset employed consisted of the ellagitannin concentration determined in the wines during the ageing period (from sampling points 1 to 9; Table 1).

Statistical differences for the concentration of ellagitannin and individual ellagitannins in each wine were determined by one-way ANOVA and Tukey's honestly significant difference test. For the study of the vescalagin/castalagin ratio and its evolution in the wines aged in the three types of barrel, the results of both wines (V1 and V2) were considered together $(n=4)$. Data were processed with the sPSS 13.0 software package (SPSS, Chicago, IL, USA).

\section{Results and discussion}

\section{Ellagitannin concentration}

The evolution of the ellagitannin concentration in all wine samples (wines V1 and V2 aged in new and second-fill French oak barrels and new American oak barrels) during winemaking and ageing is shown in Figure 2 (for further details see also Tables S1, S2). The concentration of ellagitannin showed the same evolution over time independently of the botanical origin of the oak wood or the age of the barrels used. In all cases, the ellagitannin concentration increased initially and reached a maximum after approximately 3 months in oak barrels (sampling point 3). From this time onwards, the concentration decreased in all the types of samples. Similar results were obtained in a study by Jourdes et al. (2011) in wines aged in French oak barrels, despite the fact that MLF occurred in tanks

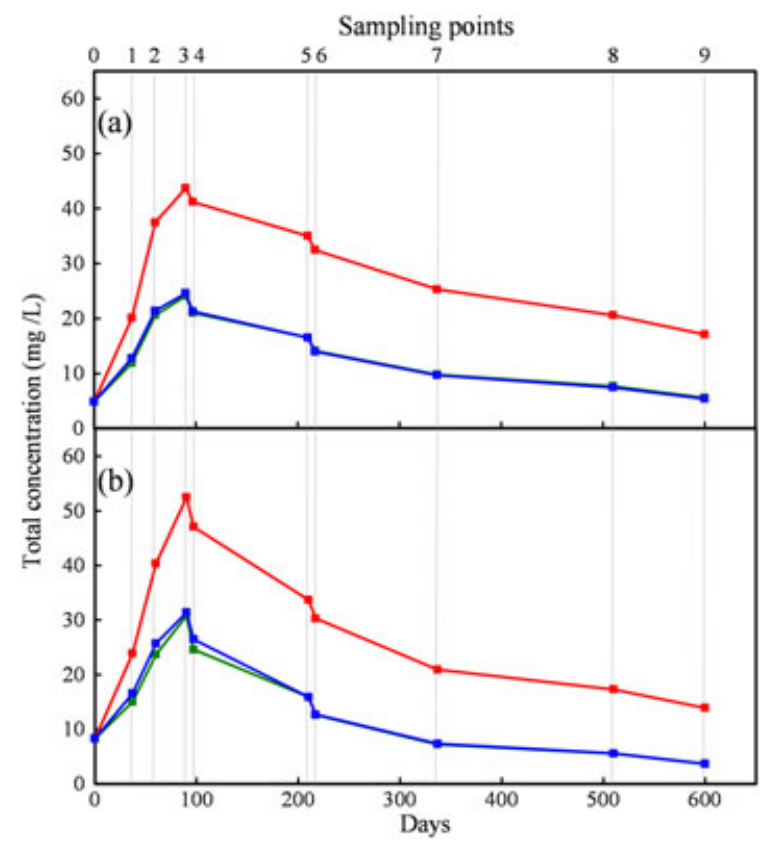

Figure 2. Evolution of the ellagitannin concentration in wines (a) V1 and (b) V2

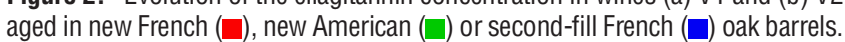

(Jourdes et al. 2011) and in barrels in the current work, which suggests that the time when maximum concentration of ellagitannin is reached is determined by the residence time in barrels. Furthermore, because our study was carried out under commercial winery conditions, the influence of the rackings on the ellagitannin concentration could be assessed. Although there was only 7 days between sampling points 3 and 4 and between sampling points 5 and 6 , there was a significant decrease in the ellagitannin concentration between the sampling points (Figure 2). The wines were racked between both sets of sampling points. The first sampling points of each pair (sampling points 3 and 5) correspond with the time before racking and the second (sampling points 4 and 6) correspond to 7 days after the racking. Racking causes a significant entrance of oxygen into the wine, and as a consequence, oxidation reactions can be more important after this time. Thus, after racking, ellagitannin can be more easily oxidised, and consequently, the decrease in their concentration is greater than might be expected.

Significance of the type of oak barrel. As for the botanical origin of wood, comparison was carried out between wines aged in new French (F) and new American (A) oak barrels (Figure 2). As indicated earlier, the evolution profiles of the ellagitannin concentration in the wines were similar regardless of the type of oak wood. Nevertheless, differences in the ellagitannin concentration were observed between them. The concentration was much higher in the wines aged in $\mathrm{F}$ barrels than in those aged in A barrels. At the time of maximum concentration, the concentration in the wines aged in $F$ barrels was almost twofold higher than that in the wines aged in $\mathrm{A}$ barrels. This difference can be explained by the influence of the botanical origin on the concentration of ellagitannin in oak heartwood: French oak has a concentration of ellagitannins between twofold and fourfold higher than that of American oak (Chatonnet and Dubourdieu 1998, Fernández de Simón et al. 2003, Glabasnia and Hofmann 2006, Prida and Puech 2006). The difference in the concentration between wines aged in F and A barrels, however, was lower than could 
be expected from that in wood. It should be considered that the different wood anatomy of French and American oaks conditions the process employed in the manufacture of barrels, which, in turn, conditions the degree of penetration of wine into the wood staves of the barrels, which is higher with barrels made from American oak (Zamora 2003). Hence, this smaller difference between wines aged in A barrels and those aged in F barrels might be explained by a greater penetration of wine in American oak, which would lead to a higher extraction, thus making up the lower concentration of ellagitannin in this oak.

A difference in the concentration of ellagitannin was also observed between the wines aged in French oak barrels that differ in the use [new (F) and second-fill (S)]. The wines aged in $S$ barrels showed a concentration between 1.5-fold and twofold lower than that observed in the wines aged in $\mathrm{F}$ barrels. As a result of the use of the barrels in successive years, the phenolic substances (Rous and Alderson 1983) and, in particular, the ellagitannin (Vivas and Saint-Cricq de Gaulejac 1998) from the outermost layers of the wood could be partially depleted, thus explaining this difference in the concentration. The use of barrels for wine ageing in a first vintage causes, according to our results, a reduction of nearly one-half in the amount of ellagitannin that can be released from these barrels if they are used for wine ageing in a second vintage.

Other differences related to the type of oak barrel employed during ageing can be also observed among the different types of samples during the phase when ellagitannin declines. For example, the decrease in the concentration due to the first racking is slightly higher in wines aged in A and S barrels than in wines aged in $\mathrm{F}$ barrels. If we consider these losses in relation to their maximum concentration (i.e. the proportion of the maximum concentration that is lost during the first racking), it can be observed that in the wines aged in $\mathrm{F}$ barrels, about $8 \%$ of the total concentration is lost during the first racking whereas about 15 and $16 \%$ of their maximum concentration is lost in wines aged in $\mathrm{S}$ and $\mathrm{A}$ barrels, respectively (Tables S3, S4).

At the end of the study, the wines aged in F barrels showed a higher proportion of ellagitannin in relation to the maximum concentration (on average, about 33\%) than wines aged in S and A barrels, which showed a similar proportion (about $17 \%$ on average). Thus, on the basis of the highest maximum concentration, it appears that $\mathrm{F}$ barrels were able to release a greater concentration of ellagitannin and probably during a longer period of time than the other types of barrels employed in this study, because, at the end of the study, both the concentration and the proportion of the maximum concentration of ellagitannin in these wines were higher than that in wines aged in $\mathrm{S}$ and A barrels.

The tank used for the alcoholic fermentation impacted the initial concentration of ellagitannin (Figure 2), because wine V2, which was fermented in a new oak tank, contained a higher concentration compared with that of wine Vl, which was fermented in a second-fill tank. This initial difference, however, was reduced during barrel ageing, and at the end of the study, wine V2 had a concentration of ellagitannin significantly lower than that in wine Vl (Figure 2). Current studies in our laboratory (data not shown) indicate that solutions containing one ellagitannin are more stable than solutions containing a mixture of ellagitannins. Hence, the fact that wine V2 had a higher concentration of ellagitannin might imply a higher reactivity of these compounds, which could, in turn, explain the greater losses in these wines.

\section{Individual ellagitannins}

The concentration of individual ellagitannins was monitored over the course of the study (Figure 3, Table S1). Castalagin and vescalagin were the most abundant ellagitannins (on average about 50 and $30 \%$ of the ellagitannin concentration, respectively). Grandinin and roburin E represented, on average, only about 10 and $8 \%$ of the ellagitannin concentration, respectively. This accords with the proportion that they represent in oak wood (Fernández de Simón et al. 1999, Glabasnia and Hofmann 2006, Prida and Puech 2006).

Similar to the concentration of ellagitannin, that of the individual ellagitannins in wine increased during the first 3 months in the barrels and they began to decrease just after the first racking. Some differences among the ellagitannins were observed during the phase when ellagitannin increases, which might be related to differences in extractability. Some extractability patterns that we observed in model systems (García-Estévez et al. 2015) could also be seen in the wines of the present work, despite the different conditions under which the ellagitannins were extracted. For example, the higher extractability of vescalagin in relation to castalagin and the lower extractability of grandinin and roburin $\mathrm{E}$ in relation to the other two (García-Estévez et al. 2015) were clearly observable in the present study after 1 month in the barrel (sampling point 1). This was assessed by calculating for each ellagitannin the proportion of its maximum concentration (reached at sampling point 3) during the phase where they were increasing (Tables S3, S4). Whereas at sampling point 1 , almost $60 \%$ of the maximum concentration of vescalagin was already detectable in all wines, only about $50 \%$ of the maximum concentration of castalagin and about $40 \%$ of that

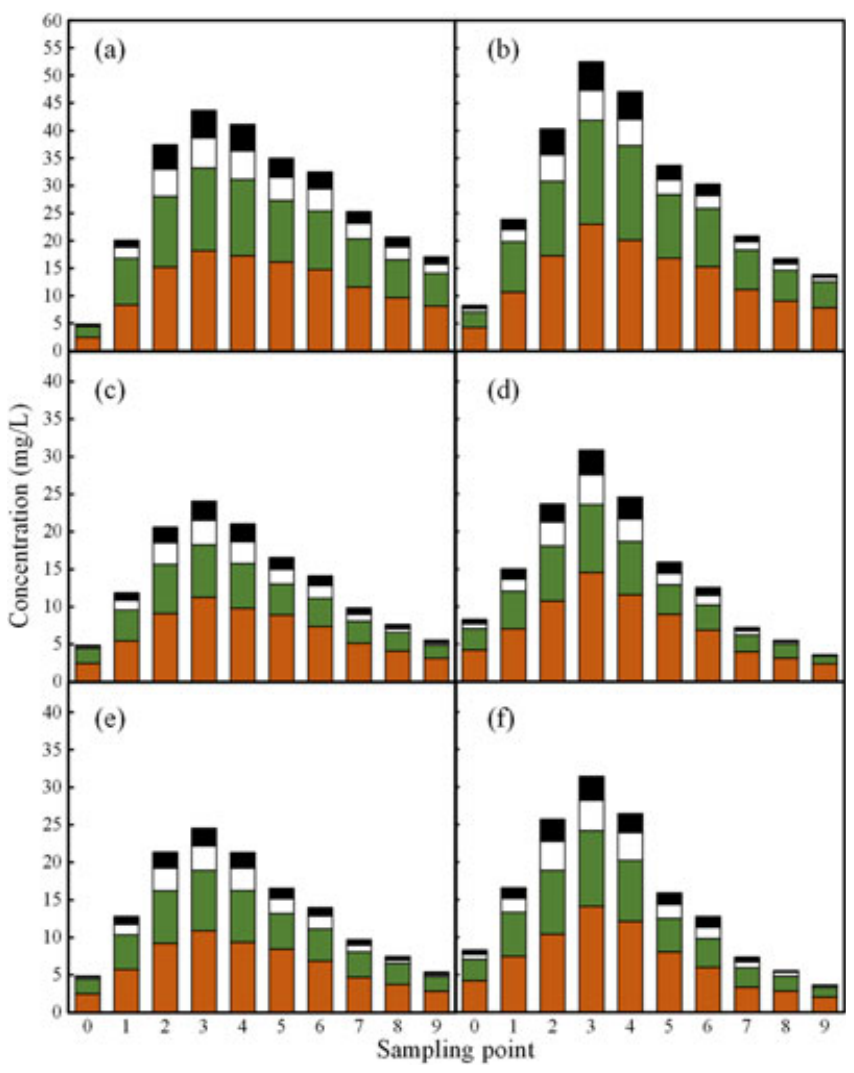

Figure 3. Concentration of individual ellagitannins [castalagin ( $\square$ ), vescalagin $(\square)$, grandinin $(\square)$ and roburin $E(\square)$ ] at the different sampling points of the $(a, c, e)$ wines $V 1$ and $(b, d, f)$ wines V2 aged in $(a, b)$ new French, $(c, d)$ new American or $(e, f)$ second-fill French oak barrels. 
of grandinin and roburin E were detected. At sampling point 2 ( 2 months in the barrels), the differences among ellagitannins were reduced (between 75 and $90 \%$ of the maximum concentration was already extracted depending on the compound and the type of wine), which might be related to the higher relative contribution of the diffusion step in the extraction process in the case of grandinin and roburin $\mathrm{E}$ (García-Estévez et al. 2015). Racking caused a reduction in the concentration of all ellagitannins, but there were no clear differences on how the individual ellagitannins were affected. In contrast, differences among the behaviour of the main oak ellagitannins have been observed during the phase where they decrease. Castalagin and vescalagin appear to be the most stable ellagitannins over time, with the higher proportion of their maximum concentration at the end of the study (Tables S3, S4). To be precise, castalagin showed the highest proportion ranging from 44.5 to $14.6 \%$ in all wines (depending on the type of oak barrel). At the end of the study, the proportion of the maximum vescalagin concentration (ranging from 39.9 to $11.5 \%$ ) was slightly lower than that for castalagin. The proportion of grandinin and roburin E was much lower than that observed for castalagin and vescalagin in all wines. In fact, in the wines aged in A barrels coming from wine V2, these compounds showed at the end of the study about $2 \%$ of their maximum concentration. This fact points to a possible higher trend of these glycosidic compounds to be oxidised and/or hydrolysed in relation to that for vescalagin and castalagin.

Because of these differences in extractability and reactivity of the different ellagitannins, significant changes in the proportion of the ellagitannins analysed in this study were observed. The most striking change in the proportion was observed between sampling points 0 (devatting) and 1 (1 month of stay in the barrels). After the stay of the wine in the oak tank, grandinin and roburin $\mathrm{E}$ each represented, on average, about $6 \%$ of the ellagitannin concentration whereas during the stay in the oak barrels they reached approximately $12-14 \%$. The difference in the surface-to-volume ratio of the tanks compared with that of the barrels along with a possible easier extractability of castalagin and vescalagin compared with that of grandinin and roburin E might explain these changes in the proportion of the individual ellagitannins. Thus, the higher surface-to-volume ratio of the barrels in relation to that of the tank has probably favoured a higher extraction of these two compounds once the wine is transferred to the barrels.

Significance of the type of oak barrel. The composition of the individual ellagitannins varied between the wines aged in the three types of oak barrels. The wines aged in F barrels showed at all sampling points the highest concentration for each individual ellagitannin, whereas the concentration of each individual ellagitannin was similar in wines aged in A and S barrels. As occurred for the concentration of ellagitannin, differences were observed in the stability of the individual ellagitannins among the three types of barrels. The proportion of all the ellagitannins at the end of the study as a function of their maximum concentration was always higher in the wines aged in $\mathrm{F}$ barrels compared with that in wines aged in $\mathrm{A}$ or $\mathrm{S}$ barrels, in which, in turn, the proportion was quite similar (Tables S3, S4). For example, castalagin, whose proportion in relation to its maximum concentration was the highest at the end of the study, showed a higher proportion in the wines aged in $\mathrm{F}$ barrels (on average, around 39\%) than that observed in the wines aged in A or S barrels (on average, about $21 \%$ ). Nevertheless, although the concentration and stability of the individual ellagitannins were similar in wines aged in A and S barrels, the vescalagin/castalagin ratio varied between wines aged in French oak barrels (both new and second-fill) and those aged in American oak barrels. Table 2 shows the global value of this ratio for the wines aged in F, A and S barrels, calculated as the mean of all the ratios determined in each type of wine and in both wines (wines V1 and V2) during ageing (from sampling points 1-9). The vescalagin/castalagin ratio was significantly higher in the wines aged in French oak barrels (both new and second-fill) than that in wines aged in A barrels. Thus, the determination of this ratio in barrel-aged wines can provide relevant information on the type of oak, French or American, from which the barrel has been made. As the concentration of ellagitannin in wines depends on their level in oak wood, it would be interesting to know if this ratio is also higher in French oak wood than that in American oak wood. Because this is the first time, however, that such a ratio has been proposed for comparative purposes, no data on oak wood are available. Nevertheless, it can be calculated from the quantitative data reported in studies of oak wood; for example, from the concentration reported by Prida and Puech (2006) in French and American oak wood, it can be seen that French oak wood has a higher vescalagin/castalagin ratio than that of American oak, which is in accordance with the ratio observed in the wine samples of our study. In contrast, in other studies with oak wood (Fernández de Simón et al. 1999, Glabasnia and Hofmann 2006), the relationship between this calculated ratio and the botanical origin is not as clear as in the study of Prida and Puech (2006). It has to be taken into account, however, that the concentration in wood is not the only factor affecting the concentration of ellagitannin in wines and, consequently, the vescalagin/castalagin ratio. Other factors directly conditioned by the botanical origin of the wood can exert a significant influence on this ratio. Among them, it is important to note that it has recently been demonstrated that barrels made from American oak allow a greater penetration of oxygen into the barrels during wine ageing and a higher initial oxygen contribution (Del Álamo-Sanza

Table 2. Effect of ageing wines in new French, new American and second-fill French oak barrels on the mean vescalagin/castalagin ratio.

\begin{tabular}{|c|c|c|c|}
\hline \multirow{5}{*}{$\begin{array}{l}\text { Sampling } \\
\text { point } \neq\end{array}$} & \multicolumn{3}{|c|}{ Mean global ratio† } \\
\hline & F barrelsł & A barrelsł & $S$ barrelsł \\
\hline & $0.76 \pm 0.08 a$ & $0.58 \pm 0.03 b$ & $0.70 \pm 0.07 a$ \\
\hline & \multicolumn{3}{|c|}{ Mean ratio at different sampling points† } \\
\hline & F barrels & A barrels & $S$ barrels \\
\hline 1 & $0.93 \pm 0.09 a$ & $0.74 \pm 0.03 b$ & $0.80 \pm 0.0 \mathrm{lb}$ \\
\hline 2 & $0.81 \pm 0.04 a$ & $0.70 \pm 0.02 b$ & $0.79 \pm 0.04 a$ \\
\hline 3 & $0.82 \pm 0.02 \mathrm{a}$ & $0.62 \pm 0.01 \mathrm{c}$ & $0.73 \pm 0.0 \mathrm{lb}$ \\
\hline 4 & $0.83 \pm 0.03 a$ & $0.61 \pm 0.01 \mathrm{c}$ & $0.70 \pm 0.04 \mathrm{~b}$ \\
\hline 5 & $0.69 \pm 0.01 \mathrm{a}$ & $0.45 \pm 0.02 \mathrm{c}$ & $0.56 \pm 0.0 \mathrm{lb}$ \\
\hline 6 & $0.71 \pm 0.02 \mathrm{a}$ & $0.50 \pm 0.02 c$ & $0.63 \pm 0.0 \mathrm{lb}$ \\
\hline 7 & $0.70 \pm 0.06 a$ & $0.57 \pm 0.02 b$ & $0.74 \pm 0.04 a$ \\
\hline 8 & $0.65 \pm 0.04 a$ & $0.60 \pm 0.01 \mathrm{~b}$ & $0.72 \pm 0.04 a$ \\
\hline 9 & $0.67 \pm 0.08 \mathrm{a}$ & $0.49 \pm 0.06 \mathrm{~b}$ & $0.65 \pm 0.03 a$ \\
\hline
\end{tabular}

†Mean value and standard deviation $(x \pm S D)$. Different lower case letters within each row indicate a significant difference $(n=36$ for the global ratio; $n=4$ for the mean ratio at different stages, $P<0.05)$. $\ddagger$ For further information about sampling points, see Table 1. A, new American oak; F, new French oak; S, second-fill French oak. 
and Nevares 2014). Moreover, in a simultaneous study carried out in our laboratory in wine-like model systems containing oak chips and different concentration values of dissolved oxygen (data not shown), vescalagin was more sensitive towards oxygen than castalagin during the extraction phase. Consequently, in the barrels made from American oak wood, a higher concentration of oxygen would cause a greater decrease in the concentration of vescalagin and, therefore, a decrease of the vescalagin/castalagin ratio. Thus, the differences observed in the vescalagin/castalagin ratio in wines aged in oak wood barrels are doubly conditioned by the botanical origin of the wood employed in the barrels. In addition, the anatomy of the oak, French or American, determines the method used in oak cooperage, which in turn conditions the degree of penetration of wine and the rate of the wood degassing process, which are both higher in American than in French wood (Del Álamo-Sanza and Nevares 2014). Consequently, the method used in cooperage enhances the differences because of the botanical origin and, thus, the oxygen transfer into the barrels, which directly affects the vescalagin/castalagin ratio.

The evolution of this ratio in the different types of wines during barrel ageing can also be observed in Table 2 . This ratio slightly decreased from the beginning to the end of the study in all wines, which is indicative of a greater loss of vescalagin in relation to castalagin. This result also confirms the higher stability of castalagin in wines compared with that of other ellagitannins (Jourdes et al. 2011). In addition, it can be seen that at all stages of barrel ageing, wines aged in F barrels show a ratio significant higher than that of wines aged in A barrels, independently of whether new or second-fill barrels. The only exception can be found at sampling point 1 , where wines aged in $\mathrm{F}$ and $\mathrm{S}$ barrels show higher values than wines aged in $\mathrm{A}$ barrels, but the difference between $\mathrm{S}$ and $\mathrm{A}$ wines is not significant. This similarity between $\mathrm{S}$ and $\mathrm{A}$ wines might be because this sampling point is shortly after devatting and because the tank where alcoholic fermentation of all wines was carried out was made of French oak wood. As previously indicated, the ellagitannin composition in these early stages in the barrel is conditioned by the stay in the tank. This means that the effect of the tank on the ellagitannin composition of the wine cannot be neglected. Factors such as the type of tank (new or second-use, botanical origin) and the length of stay in it can affect not only the total amount of ellagitannin in wines but also the proportion of the different ellagitannins in the wine. Nevertheless, as the wines stay in the barrels, the extraction of the ellagitannin from them occurs and, then, the ellagitannin composition of the wine begins to be conditioned by the barrel. Consequently, the influence of the type of oak used in the tank is gradually lessened.

\section{Discriminant analysis}

The influence of the oak type of the barrels on the ellagitannin composition of the wines was evaluated by means of discriminant analysis, which was performed as a supervised pattern recognition method so as to allocate the wine samples to their wine group (wines aged in F barrels, wines aged in A barrels and wines aged in S barrels). Discriminant analysis is a statistical technique widely used to investigate differences between groups on the basis of the attributes of the samples, indicating which attributes contribute most to group separation (Brereton 2003). The three groups were defined and ten variables (ellagitannin concentration, the concentration of the four individual ellagitannins, the proportion of the ellagitannin concentration of the four individual ellagitannins and the vescalagin/castalagin ratio) with the dataset from both wines (wine Vl and wine V2) were used to carry out the discriminant analysis (54 sets of data). As a result, two discriminant functions were built using eight variables. The castalagin concentration and the proportion of roburin E were excluded by the algorithm, because they did not significantly contribute to the classification. The discriminant analysis results are shown in Figure 4. Factor 1 allowed the discrimination of wines aged in $\mathrm{F}$ barrels from the remaining samples, whereas it could be possible to discriminate along the factor 2 between samples of wines aged in new oak barrels (both $\mathrm{F}$ and $\mathrm{A}$ ) and those aged in $S$ barrels. Hence, three groups were formed, each one corresponding to a group of samples of wines aged in the same type of oak barrels, with a slight overlapping between $\mathrm{F}$ barrels group and $\mathrm{S}$ barrels group and between A barrels group and $\mathrm{S}$ barrels group.

The standardised canonical discriminant factor coefficients for each variable were used to evaluate their significance to each function. Coefficients with large absolute values correspond to variables with greater discriminating ability. The variables with the highest contribution on factor 1 were ellagitannin concentration, vescalagin concentration and the vescalagin/castalagin ratio (coefficients: $5.060,-1.452$ and 1.284, respectively). Therefore, the higher ellagitannin concentration in wines aged in $\mathrm{F}$ barrels allowed the separation of this group from A and S groups along factor 1 . The most important variables on factor 2 were vescalagin proportion, vescalagin/castalagin ratio and vescalagin concentration (coefficients: $2.886,-2.592$ and 1.447, respectively). Thus, wines aged in A barrels were grouped separately from wines aged in S barrels along factor 2, mainly as a consequence of the difference in the vescalagin concentration.

Although the final purpose of this work was not to develop a methodology to classify different wine samples into predefined groups, discriminant analysis can also be used to classify samples into groups, showing how well the function separates the groups. This statistical technique builds an equation, which will minimise the possibility of misclassifying the samples into their respective groups or categories (Brereton 2003). Table 3 shows the results from the leaveone-out cross-validation classification and external validation. Leave-one-out cross-validation was performed directly from the discriminant analysis carried out with the whole data set. External validation was performed using the data from wine

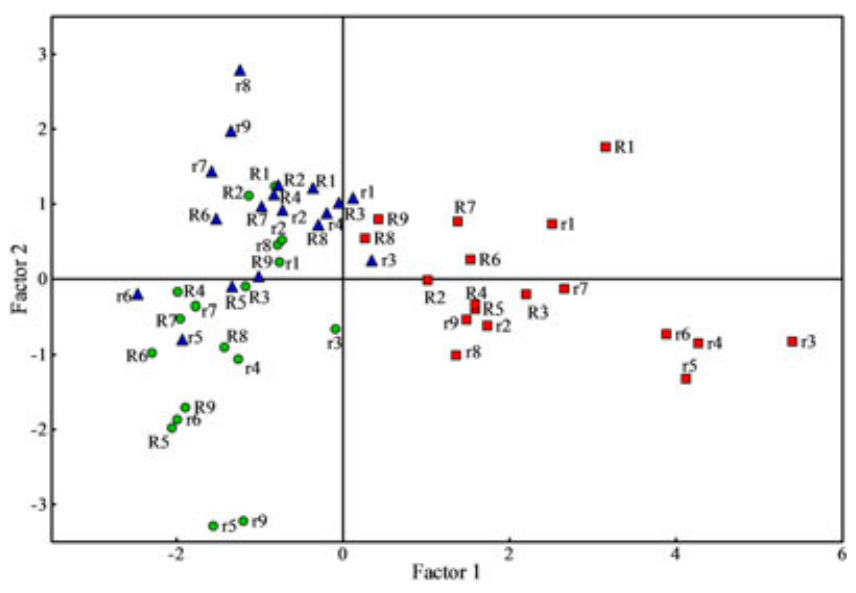

Figure 4. Discriminant analysis plot of data from wines aged in new French ( $\square$ ), new American (O) and second-fill French $(\mathbf{\Delta})$ oak barrels. R, wine V1; r, wine V2. The numbers correspond to the sampling points (Table 1). 
Table 3. Classification results obtained in the one-leave-out cross and external validations of the discriminant analysis model.

\begin{tabular}{|c|c|c|c|c|c|}
\hline & \multirow[t]{2}{*}{ Original group } & \multicolumn{3}{|c|}{ Predicted group membership } & \multirow[t]{2}{*}{ Tota } \\
\hline & & A barrels & F barrels & S barrels & \\
\hline \multicolumn{6}{|c|}{ One-leave-out cross-validation } \\
\hline \multirow[t]{3}{*}{ Count } & A barrels & 13 & 0 & 5 & 18 \\
\hline & F barrels & 0 & 16 & 2 & 18 \\
\hline & S barrels & 4 & 1 & 13 & 18 \\
\hline \multirow[t]{3}{*}{ Proportion (\%) } & A barrels & 72.2 & 0 & 27.8 & 100 \\
\hline & F barrels & 0 & 88.9 & 11.1 & 100 \\
\hline & S barrels & 22.2 & 5.6 & 72.2 & 100 \\
\hline \multicolumn{6}{|c|}{ External validation (V2 samples in the model built from V1 samples) } \\
\hline \multirow[t]{3}{*}{ Count } & A barrels & 5 & 0 & 4 & 9 \\
\hline & F barrels & 0 & 9 & 0 & 9 \\
\hline & $\mathrm{S}$ barrels & 3 & 1 & 5 & 9 \\
\hline \multirow[t]{3}{*}{ Proportion $(\%)$} & A barrels & 55.6 & 0 & 44.4 & 100 \\
\hline & F barrels & 0 & 100 & 0 & 100 \\
\hline & S barrels & 33.3 & 11.1 & 55.6 & 100 \\
\hline \multicolumn{6}{|c|}{ External validation (V1 samples in the model built from V2 samples) } \\
\hline \multirow[t]{3}{*}{ Count } & A barrels & 6 & 0 & 3 & 9 \\
\hline & F barrels & 0 & 7 & 2 & 9 \\
\hline & S barrels & 2 & 1 & 6 & 9 \\
\hline \multirow[t]{3}{*}{ Proportion $(\%)$} & A barrels & 55.6 & 0 & 44.4 & 100 \\
\hline & F barrels & 0 & 77.8 & 22.2 & 100 \\
\hline & S barrels & 22.2 & 11.1 & 66.7 & 100 \\
\hline
\end{tabular}

A, new American oak; F, new French oak; S, second-fill French oak barrels.

$\mathrm{V} 1$ in the model built from the data of wine V2 and vice versa. From cross-validation, $77.8 \%$ of the samples were correctly classified. With the $\mathrm{F}$ barrels group, the results were satisfactory, with $88.9 \%$ of samples correctly classified. The samples with the highest proportion of misclassification were those aged in A barrels $(72.2 \%$ of the samples were correctly classified). The classification mistakes for $\mathrm{F}$ barrels and A barrels groups consisted in a misclassification into the $\mathrm{S}$ barrels group. Thus, no samples from the $\mathrm{F}$ barrels group were misclassified into the A barrels group, and neither the reverse. Of the samples of the wines aged in S barrels, $72.2 \%$ were correctly classified. Results from external validation are similar, and no samples from the F barrels group were classified in the A barrels group and the reverse. The proportion of misclassification, however, is a little higher for samples from A and S barrels than in cross-validation, probably because of the low number of samples implied in building the classification models. In all cases, classification showed that the $\mathrm{S}$ barrels group appeared to be an intermediate group between those formed by the samples of wines aged in new barrels. This could be a consequence of the low concentration of ellagitannin, similar to that observed in the wines aged in A barrels, and of the higher vescalagin concentration, similarly to wines aged in $\mathrm{F}$ barrels. Most of the misclassified samples corresponded to the early sampling points. Ellagitannin concentration at these sampling points is still not significant enough to allow a clear differentiation.

\section{Conclusions}

The present study has shown that the type of oak barrel employed for ageing has a strong influence on the ellagitannin profile of the wines aged in them. As a result, such influence cannot be ignored, considering the role that these compounds can play in colour stabilisation of red wines (Vivas and Glories 1996), in the formation of new anthocyano-ellagitannin pigments (Chassaing et al. 2010, García-Estévez et al. 2013) and in wine astringency depending on their concentration (Glabasnia and Hofmann 2006, Stark et al. 2010, Michel et al. 2011, Chira and Teissedre 2013), as well as the different chemical and biological activities that they exhibit (Quideau et al. 2005, Jourdes et al. 2009).

The ellagitannin concentration might be useful in differentiating wines aged in new French barrels from those aged in new American or in second-fill French oak barrels, whereas the vescalagin/castalagin ratio might assist in tracing the oak type (French or American) of the barrels used for ageing. This ratio is doubly conditioned by the botanical origin of the oak employed in the barrel. First, by the concentration and proportion of the ellagitannins in the oak wood and second, by the different permeability of the staves to oxygen, which is a consequence of the method employed in cooperage and, in turn, a direct consequence of the wood anatomy. The present study has also shown that discriminant analysis applied to the ellagitannin concentration data differentiates the oak type of the barrels used for ageing.

\section{Acknowledgements}

This work was supported by the Spanish MINECO and FEDER (Project ref. AGL2014-58486-C2-1-R). The authors also thank Bodegas La Horra (La Horra, Burgos, Spain) for supplying the wine samples.

\section{References}

Brereton, R.G. (2003) Chemometrics: data analysis for the laboratory and chemical plant (John Wiley \& Sons: Chichester, England).

Cadahía, E., Varea, S., Muñoz, L., Fernández de Simón, B. and GarcíaVallejo, M.C. (2001) Evolution of ellagitannins in Spanish, French, and American oak woods during natural seasoning and toasting. Journal of Agricultural and Food Chemistry 49, 3677-3684.

Chassaing, S., Lefeuvre, D., Jacquet, R., Jourdes, M., Ducasse, L. Galland, S., Grelard, A., Saucier, C., Teissedre, P.L., Dangles, O. and 
Quideau, S. (2010) Physicochemical studies of new anthocyanoellagitannin hybrid pigments: about the origin of the influence of oak $C$-glycosidic ellagitannins on wine color. European Journal of Organic Chemistry 2010, 55-63.

Chatonnet, P. and Dubourdieu, D. (1998) Comparative study of the characteristics of American white oak (Quercus alba) and European oak (Quercus petraea and Q. robur) for production of barrels used in barrel aging of wines. American Journal of Enology and Viticulture 49, 79-85.

Chira, K. and Teissedre, P.L. (2013) Extraction of oak volatiles and ellagitannins compounds and sensory profile of wine aged with French winewoods subjected to different toasting methods: behaviour during storage. Food Chemistry 140, 168-177.

Chira, K., Zeng, L., Le Floch, A., Péchamat, L.P., Jourdes, M. and Teissedre, P.L. (2015) Compositional and sensory characterization of grape proanthocyanidins and oak wood ellagitannin. Tetrahedron 71, 2999-3006.

Del Álamo-Sanza, M. and Nevares, I. (2014) Recent advances in the evaluation of the oxygen transfer rate in oak barrels. Journal of Agricultural and Food Chemistry 62, 8892-8899.

Fernández de Simón, B., Cadahía, E., Conde, E. and García-Vallejo, M. C. (1999) Ellagitannins in woods of Spanish, French and American oaks. Holzforschung 53, 147-150.

Fernández de Simón, B., Hernández, T., Cadahía, E., Dueñas, M. and Estrella, I. (2003) Phenolic compounds in a Spanish red wine aged in barrels made of Spanish, French and American oak wood. European Food Research and Technology 216, 150-156.

Fridrich, D., Glabasnia, A., Fritz, J., Esselen, M., Pahlke, G., Hofmann, T. and Marko, D. (2008) Oak ellagitannins suppress the phosphorylation of the epidermal growth factor receptor in human colon carcinonma cells. Journal of Agricultural and Food Chemistry 56, 3010-3015.

García-Estévez, I., Alcalde-Eon, C., Le Grottaglie, L., Rivas-Gonzalo, J. C. and Escribano-Bailón, M.T. (2015) Understanding the ellagitannin extraction process from oak wood. Tetrahedron 71, 3089-3094.

García-Estévez, I., Escribano-Bailón, M.T., Rivas-Gonzalo, J.C. and Alcalde-Eon, C. (2010) Development of a fractionation method for the detection and identification of oak ellagitannins in red wines. Analytica Chimica Acta 660, 171-176.

García-Estévez, I., Escribano-Bailón, M.T., Rivas-Gonzalo, J.C. and Alcalde-Eon, C. (2012) Validation of a mass spectrometry method to quantify oak ellagitannins in wine samples. Journal of Agricultural and Food Chemistry 60, 1373-1379.

García-Estévez, I., Jacquet, R., Alcalde-Eon, C., Rivas-Gonzalo, J.C., Escribano-Bailón, M.T. and Quideau, S. (2013) Hemisynthesis and structural and chromatic characterization of delphinidin 3-Oglucoside-vescalagin hybrid pigments. Journal of Agricultural and Food Chemistry 61, 11560-11568.

Glabasnia, A. and Hofmann, T. (2006) Sensory-directed identification of taste-active ellagitannins in American (Quercus alba L.) and European oak wood (Quercus robur L.) and quantitative analysis in bourbon whiskey and oak-matured red wines. Journal of Agricultural and Food Chemistry 54, 3380-3390.

Hofmann, T., Glabasnia, A., Schwarz, B., Wisman, K.N., Gangwer, K.A. and Hagerman, A.E. (2006) Protein binding and astringent taste of a polymeric procyanidin, 1,2,3,4,6-penta- $O$-galloyl-beta- $D$-glucopyranose, castalagin, and grandinin. Journal of Agricultural and Food Chemistry 54, 9503-9509.

Jourdes, M., Lefeuvre, D. and Quideau, S. (2009) C-Glycosidic ellagitannins and their influence on wine chemistry. Quideau, S., ed. Chemistry and biology of ellagitannins: an underestimated class of bioactive plant polyphenols (World Scientific Publishing: Singapore) pp. 320-365.

Jourdes, M., Michel, J., Saucier, C., Quideau, S. and Teissedre, P.L. (2011) Identification, amounts, and kinetics of extraction of Cglucosidic ellagitannins during wine aging in oak barrels or in stainless steel tanks with oak chips. Analytical and Bioanalytical Chemistry 401, 1531-1539.

Masson, G., Moutounet, M. and Puech, J.L. (1995) Ellagitannin content of oak wood as function of species and of sampling position in the tree. American Journal of Enology and Viticulture 46, 262-268.

Michel, J., Jourdes, M., Silva, M.A., Giordanengo, T., Mourey, N. and Teissedre, P.L. (2011) Impact of concentration of ellagitannins in oak wood on their levels and organoleptic influence in red wine. Journal of Agricultural and Food Chemistry 59, 5677-5683.

Prida, A. and Puech, J.L. (2006) Influence of geographical origin and botanical species on the content of extractives in American, French, and East European oak woods. Journal of Agricultural and Food Chemistry 54, 8115-8126.

Puech, J.L., Feuillat, F. and Mosedale, J.R. (1999) The tannins of oak heartwood: structure, properties, and their influence on wine flavor. American Journal of Enology and Viticulture 50, 469-478.

Puech, J.L., Feuillat, F., Mosedale, J.R. and Puech, C. (1996) Extraction of ellagitannins from oak wood of model casks. Vitis 35, 211-214.

Quideau, S., Jourdes, M., Lefeuvre, D., Montaudon, D., Saucier, C., Glories, Y., Pardon, P. and Pourquier, P. (2005) The chemistry of wine polyphenolic C-glycosidic ellagitannins targeting human topoisomerase II. Chemistry. A European Journal 11, 6503-6513.

Rous, C. and Alderson, B. (1983) Phenolic extraction curves for white wine aged in French and American oak barrels. American Journal of Enology and Viticulture 34, 211-215.

Scalbert, A., Monties, B. and Favre, J.M. (1988) Polyphenols of Quercus robur: adult tree and in vitro grown calli and shoots. Phytochemistry 27, 3483-3488

Stark, T., Wollmann, N., Wenker, K., Loesch, S., Glabasnia, A. and Hofmann, T. (2010) Matrix-calibrated LC-MS/MS quantitation and sensory evaluation of oak ellagitannins and their transformation products in red wines. Journal of Agricultural and Food Chemistry 58, 6360-6369.

Timberlake, C.F. and Bridle, P. (1976) Interactions between anthocyanins, phenolic compounds, and acetaldehyde and their significance in red wines. American Journal of Enology and Viticulture 27, 97-105.

Vivas, N. and Glories, Y. (1996) Role of oak wood ellagitannins in the oxidation process of red wines during aging. American Journal of Enology and Viticulture 47, 103-107.

Vivas, N. and Saint-Cricq de Gaulejac, N. (1998) Influence de la durée d'utilisation des barriques sur leurs apports aux vins. Vivas, N., ed. Actes du IV colloque des sciences et techniques de la tonnellerie (Vigne et Vin Publications Internationales: Bordeaux, France) pp. 65-74.

Zamora, F. (2003) Elaboración y crianza del vino tinto: Aspectos científicos y prácticos (Ediciones A. Madrid Vicente \& Ediciones Mundi-Prensa: Madrid, Spain).

\section{Manuscript received: 20 October 2016}

Revised manuscript received: 10 March 2017

Accepted: 13 March 2017

\section{Supporting information}

Additional Supporting Information may be found online in the supporting information tab for this article. http://onlinelibrary. wiley.com/doi/10.1111/ajgw.12292/abstract

Table S1. Effect of ageing on the concentration of individual ellagitannins and of ellagitannin in wine V1.

Table S2. Effect of ageing on the concentration of individual ellagitannins and of ellagitannin in wine V2.

Table S3. Proportion of the individual and total ellagitannin concentration in relation to their corresponding maximum concentration in wine Vl.

Table S4. Proportion of the concentration of individual ellagitannins and ellagitannin in relation to their corresponding maximum concentration in wine V2. 